\title{
Etnomatemáticas en Artesanías de Trenzado: un modelo metodológico para investigación
}

\author{
Ethnomathematics in Braiding Crafts: \\ a methodological model for research
}

\author{
María Luisa Oliveras* \\ Veronica Albanese**
}

\begin{abstract}
Resumen
El área temática del Proyecto de Investigación, parte del cual exponemos en este artículo, es Etnomatemáticas. El propósito de investigación es la caracterización y valoración del conocimiento socio-cultural, implícito en la práctica diaria. En el contexto geográfico de Argentina, investigamos la matemática implícita en artesanías de trenzados, elaborando para esto un método propio de análisis etnomatemático. El instrumento metodológico MOMET que se crea para este estudio interpretativo formal de artesanías de trenzado tiene en cuenta dos aspectos: el producto final de la labor artesanal analizado en su complejidad global y el proceso que se lleva a cabo para realizarlo. La herramienta metodológica elaborada está constituida por dos componentes: un Método de análisis etnográfico (MET) y un Modelo de análisis matemático (MOM). El conjunto de los dos nos proporciona el instrumento metodológico MOMET, que permite la Modelización Etnomatemática de las artesanías de trenzado.
\end{abstract}

Palabras-Clave: Etnomatemáticas. Artesanías de Trenzados. Análisis Etnográfico. Modelización Matemática. Método de investigación.

\footnotetext{
* Doctora en Matemáticas. Universidad de Granada (UGR). Investigadora y Profesora Titular de Didáctica de la Matemática en Universidad de Granada (UGR). Facultad de Ciencias de la Educación. Granada, España. Dirección Postal: Campus Cartuja s/n 18079. Granada. España. E-mail: oliveras@ugr.es

*** Doctoranda en Educación por la Universidad de Granada (UGR), España. Facultad de Ciencias de la Educación. Dirección Postal: Campus Cartuja s/n 18079. Granada. España. E-mail: very_alba@hotmail.it
} 


\begin{abstract}
We present part of a research project from the field of Ethnomathematics. The purpose of the research is to characterize and assess the socio-cultural knowledge implicit in daily practice. The geographical context is Argentina. We investigated the mathematics implicit in braiding craftwork, producing a proper method for this ethnomathematical analysis. The methodological instrument, called MOMET, created for this "formal interpretative study" of braiding craftwork, focused on two aspects: the final product of the craftwork analysed in its overall complexity, and the process carried out to create it. The methodological tool developed consists of two components: an ethnographic analysis method (MET) and a mathematical analysis model (MOM). Together they compose the MOMET methodological tool, which enables Ethnomathematical modelling of braiding craftwork.
\end{abstract}

Keywords: Ethnomathematics. Braiding Craft. Ethnographic Analysis. Mathematical Modelling. Research Method.

\title{
1 Introducción
}

La Etnomatemática es un paradigma holístico, contextualizado en un movimiento que aglutina teoría y práctica, en los campos: epistemológico, matemático, investigativo, educativo y social. Actúa y estudia la manera en que los grupos culturales elaboran, comprenden y utilizan conceptos, estructuras o significados, que el investigador considera como matemáticos, en el desarrollo de su cultura. Los grupos están constituidos por personas que actúan en el desempeño de sus profesiones, y en la vida cotidiana, lo que les mantiene como individuos y como grupo.

Las posibilidades investigadoras de este campo fueron declaradas por D'Ambrosio en el ICME 5 (International Congress on Mathematical Education) del 1984, él marcó el interés de las cuestiones sociales y culturales en la Educación Matemática (D’AMBROSIO, 1985).

En este artículo exponemos parte de un Proyecto de Investigación, que se inserta como parte del trabajo doctoral de la segunda autora (ALBANESE, 2011), y que se propone de relacionar el entorno social con la educación matemática y en particular la artesanía con la matemática, considerando ambas como productos culturales.

La posición epistemológica que fundamenta a las Etnomatemáticas es la Cognición Situada y el Relativismo Social, que ven el conocimiento matemático ligado al contexto en que se produce y transmite. 
Desde 1998, en que se celebró el Primer Congreso Internacional de Etnomatemáticas ICEm1, en la Universidad de Granada (España), organizado por una de las autoras, María Luisa Oliveras (OLIVERAS; FERNÁNDEZ; FUENTES, 1998), el foro de debate internacional de este Grupo de Investigación ISGEM, (International Study Group on Ethnomathematics) se ha mantenido e incrementado con numerosos investigadores y profesores, que han producido y expuesto sus trabajos en los tres Congresos Internacionales siguientes, celebrándose en 2010 el último, el ICEm4 (Fourth International Conference on Ethnomathematics), en la Universidad de Touson, Maryland, (UE).

En otros congresos internacionales de educación matemática, como el ICME (especialmente en el último ICME11 de 2008), CIAEM y RELME, se ha manifestado un gran desarrollo de este campo, que incluye un panorama muy amplio de estudios etnográficos, multiculturales, epistemológicos, sociales, educacionales y de formación de agentes educativos para la educación formal y no formal.

Cabe destacar la relevancia que los aspectos multiculturales han adquirido en la sociedad europea y en la educación a nivel mundial.

Esta preocupación actual por la no exclusión de los grupos culturales minoritarios y por la interculturalidad como forma de desarrollo social, hace que nuestro núcleo temático tenga importancia desde el punto de vista de la educación, como proceso de configuración de la nueva generación de ciudadanos.

Ciudadanos que tienen inmersa dentro del conjunto de sus competencias, habilidades, conocimientos, valores y creencias, la capacidad de pensar matemáticamente de forma contextualizada, como una parte esencial de su cultura (OLIVERAS, 1996, 1999, 2006).

\section{Objeto y objetivos de la Investigación}

El propósito de esta investigación es plantear una cuestión de carácter social en relación con la ciencia: la valoración del conocimiento socio-cultural, implícito en la práctica diaria, que es desaprovechado por la ciencia y la educación.

El contexto geográfico de la investigación es Argentina.

El campo técnico en que se sitúa la investigación es la artesanía, existiendo precedentes de investigación en este campo (OLIVERAS, 1996).

Entendemos como artesanía la labor de creación o decoración, de manera predominantemente manual y artística, de objetos de utilidad práctica en la sociedad. 
Tratamos de encontrar artesanías que tengan suficiente potencial matemático para lograr aplicaciones educativas posteriores a niveles básico y técnico-profesional, conectando, así, la ciencia con el desarrollo de la población en sus competencias laborales.

Concretamente serán objeto de investigación las artesanías de trenzado, término con el que identificamos las artesanías de tejido en las cuales predomine una dimensión, cuyos productos son: cordeles, trenzas, o fajas.

Las trenzas son productos de un tipo de tejido simple que se realiza solo con las manos. Los cordeles son más complejos, involucran un mayor número de hilos, y para tejerlos, se utilizan aparatos suplementarios que permiten disponer y mantener los hilos separados en círculos o colocados sobre los lados de un cuadrado.

Las fajas se desarrollan en plano y se realizan con pequeños telares donde se posiciona la urdimbre y se teje la trama entre los hilos pares e impares de la urdimbre.

Los objetivos de la investigación son:

O.1 Describir y contextualizar artesanías de trenzados y estudiarlas, identificando los constructos matemáticos implícitos en ellas.

O.2 Crear un instrumento metodológico de análisis etnomatemático que se ajuste al interés del estudio y a la tipología específica del objeto estudiado.

La necesidad de crear un método propio nace de la especificidad del producto a analizar, dentro del campo etnomatemático, y de la dificultad de encontrar metodologías existentes para este tipo concreto de estudio.

El propósito de la investigación es realizar un estudio etnográfico y cualitativo de tipo interpretativo formal (O.1). A tal fin se presenta la necesidad de crear un instrumento metodológico de análisis que tenga en cuenta tanto el contexto cultural, como la forma propia del objeto, poniendo de manifiesto el contenido matemático inmerso en su elaboración y uso cultural, se pretende analizar algunos casos, con el objetivo a largo plazo de elaborar una aplicación en la educación, estructurada sobre los hallazgos de la investigación etnomatemática previa, en forma de Microproyectos interculturales Etnomatemáticos (OLIVERAS, 1996, 1997, 2008b).

Los objetivos específicos que se abordan en la parte del proyecto que se presenta en este artículo son: O.1.1 Elegir algunas artesanías de trenzados y describir el desarrollo del trabajo artesanal desde el punto de vista etnográfico. O.1.2 Identificar las matemáticas implícitas presentes en el producto, el cordel o trenza. O.1.3 Describir el proceso de trenzar, desde el punto de vista matemático formal. 
Para ello nos proponemos: O.2.1 Crear un método para el análisis etnográfico de la artesanía.O.2.2 Elaborar un procedimiento de análisis que, a través de la matemática formal, modelice el producto y el proceso.

En la caracterización de los objetivos específicos se considera la existencia de dos niveles de lenguajes matemáticos: el informal o implícito del contexto artesanal y el formal o explícito del contexto escolar-académico.

En el objetivo O.1.2 se quiere establecer una conexión o puente entre el objetivo O.1.1 de investigación etnográfica focalizada en el ámbito social de la microcultura artesanal, y el objetivo O.1.3 de modelización matemática propia de la microcultura académica.

La intención del objetivo O.2.1 - Crear un método para el análisis etnográfico - es la de reflejar los propósitos expresos en los O.1.1 y O.1.2, es decir por una parte documentar por escrito y gráficamente, el proceso de producción de estos productos, que por pertenecer a una microcultura primordialmente oral, y cuya transmisión es presencial, no tienen documentos escritos que expliquen todo su proceso de creación, desde los materiales empleados al uso social que tienen en cierto entorno geográfico, pasando por todo el proceso de elaboración; todo ello es de gran interés para nosotros desde el punto de vista etnográfico, ya que consideramos que estos objetos son signos culturales a través de los cuales podemos conocer una parte de las culturas ancestrales pervivientes hoy, aunque muy amenazadas por los procesos de globalización.

Paralelamente, es nuestra intención descubrir las formas de pensar de los artesanos que se inducen de sus formas de hacer y que traduciremos al lenguaje formal matemático, para poner de manifiesto la etnomatemática, o matemática viva (OLIVERAS, 2006), que actúa de forma implícita en la vida profesional de los artesanos, como parte importante de su vida social. Esta es la intención manifestada en el objetivo O.2.2, que es la de desarrollar el objetivo O.1.3.

En suma, con este detallado conjunto de objetivos lo que esperamos lograr es:

a) conocer la matemática implícita en las artesanías de trenzados y

b) elaborar para esto un método propio de análisis etnomatemático.

\section{Contexto}

Se ha recogido la información a través de una inmersión en el campo 
en dos escenarios de dos regiones geográficas y culturales distintas de Argentina.

Se ha realizado, en primer lugar, un periodo de observación no participante, en el que se ha contactado con la cultura artesanal, representada por un grupo de artesanos, y con uno de ellos muy especial, por ser, a su vez, matemático, físico y profesor universitario que, actualmente, también enseña en una Escuela de Artesanos, y es nuestro informante clave. Esta escuela y su entorno ya eran conocidos por las investigadoras y han sido visitadas en esta ocasión por una de ellas, en una observación no participante, enfrentando el rol de aprendiza de artesana y miembro de la comunidad, conviviendo en una de sus casas, y escuchando y debatiendo de forma continuada sobre la producción artesanal y su función social, que es la base de la que parte nuestra investigación.

Otro escenario del que se ha tomado información es una feria de artesanos que se realiza en Mataderos, un barrio popular de Buenos Aires, allá se conversó con los artesanos y se realizaron algunos aprendizajes de tejidos con cuero.

En la estancia de trabajo de campo realizada en la Escuela de Artesanías de Cafayate, ciudad situada en la región de Salta, noreste de Argentina (donde imparte docencia el profesor Luis Alberto Castagnolo, que ha sido nuestro informante clave), se han recibido clases y se han recopilado varios ejemplares de trenzas y cordeles, así como algunos artefactos utilizados como complementarios en el tejido artesanal de dichos objetos. Ejemplares trenzados y artefactos, junto a los trabajos previos del profesor Castagnolo, constituyen un punto de apoyo clave para nuestra modelización.

Adjuntamos algunas fotos, para que el lector pueda conocer aspectos concretos de los objetos artesanales que han sido estudiados con el método creado y aquí expuesto.

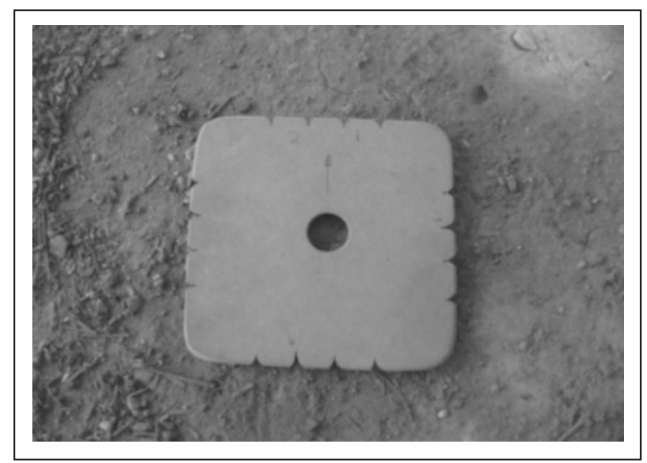

Figura 1 - La Carta 
Aparato que denominamos La carta, es un cuadrado de madera con un agujero en el centro, y unos pequeños cortes en los lados para mantener los hilos en las diversas posiciones. El Profesor Castagnolo llamaba a este aparato con el nombre marudai, y lo utilizaba situado encima de un soporte o pie (Figura 2), lo que permite trenzar cordeles con más eficacia.

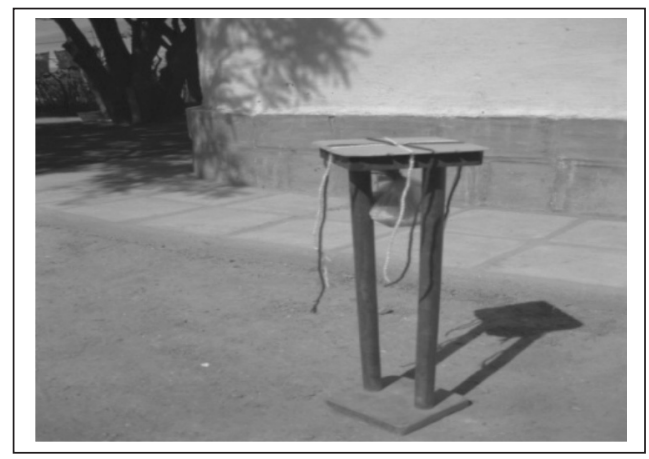

Figura 2 - La Carta en su soporte

El diseño del Lápiz lo llamamos doble rombo, también ha sido modelizado. Este cordel viene producido en modalidad de trenzado con la utilización de la carta.

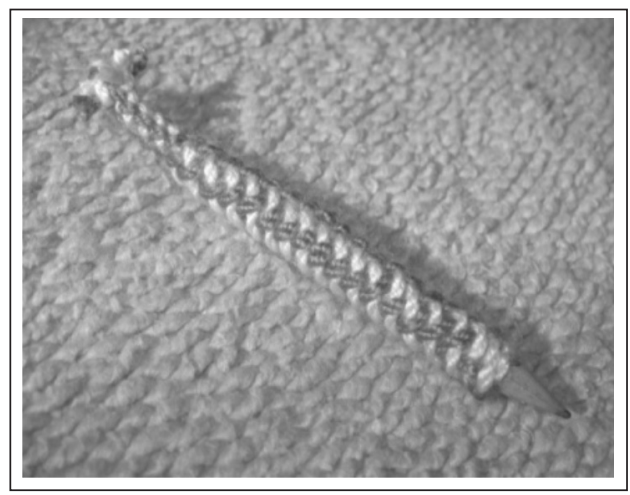

Figura 3 - Ejemplar 1 Lápiz

A este ejemplar 1 le llamamos Lápiz, porque el cordel se desarrolla alrededor de un núcleo o corazón vacío, donde la artesana 1 ubicó un lápiz. Ella era muy rápida en reconocer cualquier otro diseño que tenga esa característica. 


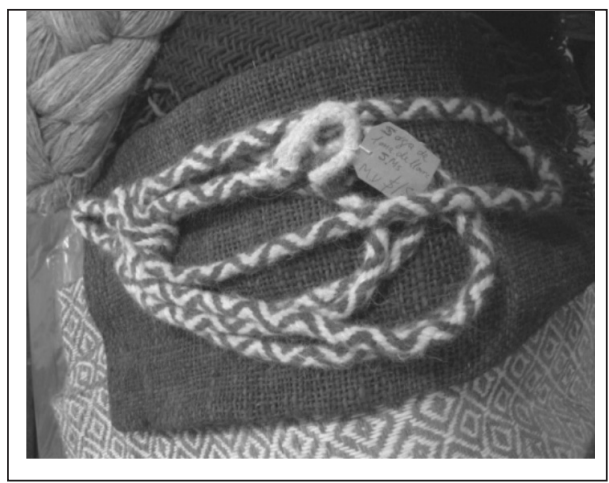

Figura 4 - Ejemplar 2 Soga

La Soga se usa para atar animales sin que se dañe su piel. Tiene de largo unos 3 metros, un grosor de 2 centímetros de diámetro, esta trenzado en hilos de lana de colores marrón y blanco.

Al Diseño de la Soga lo llamamos Estrella de 16, o de cuadrados con inversión, se realizan pequeños trozos de espirales en sentido invertido.

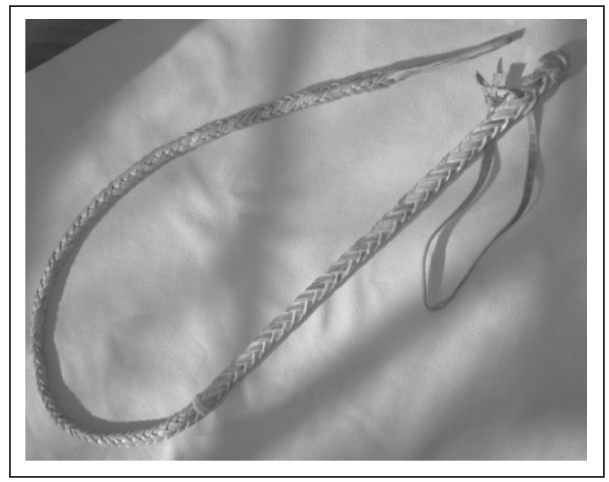

Figura 5 - Ejemplar 3 Látigo

El Látigo tiene de largo un metro y grosor de un centímetro y medio de diámetro. Es del color natural del cuero crudo, o sea marrón claro. Se usa para manejar a los animales, caballos especialmente.

Llamamos al diseño del Látigo: Trenza redonda de dos a dos, y su modelización, como la de los restantes ejemplares, ha sido muy interesante, si bien no podemos exponerla aquí por falta de espacio. Exponemos al final solo un caso de modelización matemática, el correspondiente al diseño más sencillo de los estudiados. 


\section{Cultura y microcultura}

\subsection{Cultura}

Realizamos un pequeño análisis conceptual del término Cultura, como el fundamento principal de nuestra posición epistemológica, en la que el conocimiento no es general sino que depende de la cultura.

A partir de la definición de la enciclopedia Encarta, elegida porque incluye los diversos matices que contiene el término, siguiendo su evolución epistemológica, se analiza el uso que hacen diferentes autores y se declara cual es la interpretación a la que nos referimos en nuestra investigación.

La definición del término Cultura de la enciclopedia Encarta (2009) es: cultura. (Del lat. cultûra). f. cultivo. \| 2. Conjunto de conocimientos que permite a alguien desarrollar su juicio crítico. || 3. Conjunto de modos de vida y costumbres, conocimientos y grado de desarrollo artístico, científico, industrial, en una época, grupo social, etc. $\| 4$. ant. Culto religioso. $\| \sim$ física. f. Conjunto de conocimientos sobre gimnasia y deportes, y práctica de ellos, encaminados al pleno desarrollo de las facultades corporales. $\| \sim$ popular. f. Conjunto de las manifestaciones en que se expresa la vida tradicional de un pueblo.

Analizando estas definiciones desde el punto de vista histórico se reconoce el recorrido y el desarrollo del término. De su origen latino con significado de cultivo (1), el término empieza a abarcar significados más amplios cuando Cicerone acuña la expresión cultura corpi et animi, adquiriendo, así, las matices de formación - desarrollo personal - a través de prácticas corporales (física) y logrando conocimiento para un juicio crítico (2). En el Medievo, siendo la formación estrechamente ligada al ámbito religioso, se extiende a este sentido (4). De aquí a expresar tradiciones (no solo religiosas) del pueblo se llega al significado que se considera en esta investigación por el término cultura (3) y que reinscribimos utilizando las palabras del filósofo Tylor: "cultura es aquel todo complejo que incluye el conocimiento, las creencias, el arte, la moral, el derecho, las costumbres, y cualesquiera otros hábitos y capacidades que el hombre adquiere en cuanto miembro de la sociedad." (QUINTANILLA, 1976, p. 104). En nuestro concepto de Cultura, (OLIVERAS, 1996), consideramos tres aspectos complementarios que la caracterizan, compartidos con los autores citados y son: 
1 Aspecto semiótico-cognitivo (lo que tiene que ver con símbolos, expresión, lenguaje, comunicación). Compartimos la concepción de Geertz (citado en OLIVERAS, 1996, p. 53): una cultura es un "grupo ordenado de símbolos y significantes con los que la gente construye el sentido de los hechos de su vida".

2 Aspecto socio-político (organización del trabajo, distribución del poder, relaciones sociales). Cultura es, además de los significados compartidos, las estructuras sociales y de poder, según expresa Borba (1990, p. 42): "lo que el hombre ha añadido al mundo, con el trabajo, la lucha creativa y recreativa".

3 Aspecto tecnológico (artefacto-producto), producción, para Oltra, su visión de cultura incluye "trabajos y producción creadoras, el fractal de las obras y técnicas creadas por el ser humanos" (UÑA; HERNÁNDEZ, 2004, p. 319).

\subsection{Microcultura}

Entendemos como Microcultura, una cultura restringida a un ámbito social o geográfico minoritario, no relativo a una región geográfica extensa, sino a un entorno socio-geográfico pequeño delimitado (Andalucía, en España), o bien referente a la cultura de un gremio (artesanal), o profesión (profesor, investigador), o a un grupo de edad (niños), (OLIVERAS, 2006; D’ AMBROSIO, 2008).

En el contexto de esta investigación se consideran dos microculturas: la microcultura artesanal, expresión del ámbito socio-profesional de la artesanía de trenzado, que se desarrolla en algunas regiones delimitadas de Argentina; la microcultura académica, expresión del ambiente socio-profesional constituidos por las Universidades, los investigadores y, en general, por los que se indican como científicos de impronta occidental.

El intento es crear un puente lingüístico entre la manera de expresión informal de la microcultura artesanal y el lenguaje de la microcultura académica, que indicamos como formal. La investigadora asume el rol de traductora y propone una reelaboración lingüística en términos académicos de lo que ella interpreta que ocurre en el proceso de realización del producto artesanal, a nivel de la matemática viva en el pensamiento y acción del artesano, o sentido etnomatemático de este profesional (OLIVERAS, 1996). 


\section{Fundamento teórico y enfoque de la investigación}

En las últimas dos décadas la pérdida de universalidad de las matemáticas y la consideración creciente del condicionamiento del contexto sociocultural en sus prácticas, ha dado impulso a una nueva área de investigación cuyos padres se pueden indicar en D’Ambrosio y Bishop, que hablan respectivamente de Etnomatemáticas y Enculturación Matemática. Otras investigaciones en estas temáticas son, nombrándolas entre muchas, los aportes de Ascher (1991), Gerdes (1998), Eglash (1997) y Barton (1996).

Por otra parte, se está desarrollando un creciente interés hacia la influencia de la cultura, y de las culturas, en la didáctica, en búsqueda de nuevas maneras de hacer matemáticas en las aulas (SANTILLÁN; ZACHMAN, 2009; SANDELLA, 2004). Los países latino- americanos son promotores de programas interculturales, como la Educación Intercultural Bilingüe (EIB), que nacen con el intento de responder a la demanda de diálogo y complementariedad entre la cultura de origen de la población indígena y las culturas occidentales procedentes de la migración, en nombre de una educación de la diversidad o educación inclusiva (BOLÍVAR, 2004).

Las prácticas matemáticas: contar, medir, localizar, explicar, diseñar, jugar, son herramientas indispensables para la idealización y la producción de artesanías y para lograr la traducción dela etnomatemática subyacente en ellas a otras expresiones matemáticas (D’AMBROSIO, 2008; BISHOP, 1991).

Un aporte epistemológico muy actual es el proporcionado por Vilela (VILELA, 2010) que propone, reafirmando lo dicho por Oliveras (OLIVERAS, 1996, 2006), asociar la filosofía de Wittgenstein y la etnomatemática, en la búsqueda de un punto de partida para una fundamentación teórica de las reflexiones etnomatemáticas.

Una base filosófica de la etnomatemática debe incluir y explicar la presencia de diferentes sistemas matemáticos y la coexistencia de deferentes concepciones de racionalidad. El enfoque no metafísico de Wittgenstein, que considera la matemática como un conjunto de enunciados normativos en lugar de descriptivos, es una propuesta interesante en este sentido. Hemos dicho que la etnomatemática estudia, antropológicamente, como la matemática es utilizada en prácticas específicas. Si se interpreta la matemática según la teoría de los juegos del lenguaje de Wittgenstein, resulta que no es una descripción de la realidad, pero un sistema de reglas y procedimientos. El conocimiento no es un producto único, universal y eterno, sino más bien un proceso que se desarrolla 
en la práctica, que adquiere sentido en la utilización, en la situación. A propósito del lenguaje, Wittgenstein afirma que la estructura del lenguaje estructura la realidad (Wittgenstein citado en VILELA, 2010, p. 347), es el lenguaje el que organiza la experiencia y determina los significados, es la esencia de este sistema de símbolos con reglas el que plasma la realidad. Lo que existe depende de la manera en que se expresa, se interpreta y se entiende la realidad. Los significados nacen en el uso, según Wittgenstein.

Se pueden subrayar dos aspectos de la filosofía de Wittgenstein que se acercan a la etnomatemática, uno es lo que se acaba de presentar, que el significado es inserto o incluido en las prácticas lingüísticas, ya que el lenguaje es parte del contexto en que se desarrolla el significado. El otro consiste en el aspecto no metafísico del conocimiento, que considera los significados no fijos y determinados, sino contextualizados, condición indispensable para admitir la posibilidad de que existan varias prácticas matemáticas culturalmente diferentes.

La cultura y la educación, como proceso de desarrollo de esa cultura, están muy relacionadas en nuestro marco de referencia conceptual o fundamentos, pero debemos aclarar nuestro concepto de educación.

Hay dos concepciones antipodales, o totalmente opuestas, de la educación: la escuela como un negocio, que responde a la mentalidad mercantil del neoliberalismo, y la escuela como un derecho. En el primer caso, la escuela es uno de los bienes para comprar, cuya calidad depende de las posibilidades económicas del cliente; la educación reproduce, así, la sociedad, y tiende a mantener las divisiones sociales. Aquí, las escuelas adquieren autonomía, en nombre de la calidad, y el Estado va perdiendo su rol de asegurar igualdad formal en término de cohesión y equidad (BOLÍVAR, 2004). La otra visión romántica o de la inclusión, ve la escuela como un derecho, tiende, al contrario, a integrar a la ciudadanía en unos principios y valores comunes, compatiblemente con el reconocimiento de las diferencias, con el ideal de una socialización integradora a la vez que preservadora de las identidades; tiende a hacer de todos unos incluidos sociales y llenar los vacíos de la desigualdad, se propone cambiar el orden social preestablecido (ESCUDERO; GONZALEZ; MARTINEZ, 2009).

En la perspectiva de la inclusión se identifican dos pilares sobre los cuales se basa el cambio en la concepción de la educación (TEDESCO, 2011): aprender a vivir juntos y aprender a aprender. Aprender a vivir juntos significa que la escuela debe proporcionar los valores éticos para la convivencia en una sociedad que se hace cada día más compleja y multicultural, fomentar la 
cohesión social, el dialogo, el respeto hacia el diferente. Aprender a aprender recoge el abandono de la idea de la educación como transmisión del conocimiento y la nueva necesidad de aprender cómo enfrentarse al enorme volumen de información disponible y en continua creación, de aprender a seleccionar, evaluar, asimilar y utilizar rápidamente las novedades, o sea de comprender y operar sobre el saber. Todo esto incluye la capacidad de una reconversión profesional continua que requiere un aprendizaje permanente a lo largo de toda la vida.

El rol de una escuela comprehensiva, que mire a la inclusión, es proporcionar a todos ese conjunto de conocimientos, destrezas y valores compartidos por los ciudadanos, que son necesarios para la vida en sociedad.

El interculturalismo, como política educativa y social, se preocupa de conjugar la diversidad socio-cultural y las diferencias individuales, mediante la formación en una socialización intercultural. "El currículo ha de ser rediseñado de manera que incluya también los saberes, conocimientos y valores, de la cultura originaria" (BOLÍVAR, 2004, p. 33), también en matemáticas incluyendo etnomatemáticas, o matemáticas vivas en las culturas o microculturas de los alumnos presentes en el aula, ya que el currículo de matemáticas no es neutral sino que juega un importante papel en la inclusión y en la interculturalidad de la educación (OLIVERAS, 1996, 2006, 2008a).

\section{Antecedentes del estudio}

Son antecedentes previos de este trabajo de estudio etnomatemático y educativo, en su conjunto, las investigaciones de Oliveras, en su Tesis Doctoral (OLIVERAS, 1996), y su aplicación al proyecto IDMAMIM, financiado por el programa COMENIUS de la Unión Europea, para la educación intercultural y la formación de los profesores, (OLIVERAS, 1997, 2006, 2008b).

En Argentina se encuentran varias formas de artesanías del tejido, pervivientes e históricas (FIADONE, 2003; SERVETTO et al., 1998); un ejemplo de artesanía de trenzado es el trenzado en cuero, cuyas raíces históricas se encuentran en la cultura gaucha criolla (OSORNIO, 1934; FLORES, 1960; FONTANA, 1988; FAUDONE, 2003), y tomaremos este escenario para nuestros estudios por su relevancia cultural.

Por lo que concierne el estudio de las matemáticas en los tejidos, nombramos como antecedentes: el trabajo sobre la textura de las alfombras en la artesanía andaluza de Oliveras, (OLIVERAS, 1996); la producción de cestería en el pueblo Ticuna (DA COSTA, 2009); el estudio sobre la fabricación de 
manillas por las indígenas de la etnia Ticuna (PARRA, 2003). Como antecedentes metodológicos nombramos, nuevamente, los citados de Oliveras $(1996,2006)$ y Parra (2003); el trabajo de De Bengoechea (DE BENGOECHEA, 2009), y Bolaños (BOLAÑOS, 2009). Nos interesa de manera especial el trabajo de Parra, por la parte que se refiere a la modelización matemática de la trama del tejido, ya que analiza una elaboración de tejido que, más adelante, caracterizamos como modalidad de tejido anudados. Él recoge 5 tipos de nudos y describe el diseño a través de algoritmos utilizando como lenguaje un pseudo-código de molde informático.

Subrayamos que no hemos encontrado investigaciones que traten el tema de los objetos definidos como cordeles y trenzas desde el punto de vista etnográfico y matemático.

A este propósito señalamos la Obra de los Ascher sobre los Quipu (ASCHER, 1981) por marcar la relevancia histórica que la creación de cuerdas ha tenido en las culturas andina precolombinas y por el interés, desde el punto de vista matemático, que este artefacto ha despertado, destacando, por primera vez, el potencial precisamente matemático que contiene.

Observamos que los Quipu no entran en la definición de objeto de este trabajo por no tener una dimensión dominante: aunque constituidas por cuerdas, su característica principal es la relación que une distintos tramos de cuerdas, sin embargo se piensa en la posible incorporación de los Quipu en el trabajo posterior de aplicación educativa, dentro del proyecto que constituye nuestra investigación doctoral.

Indicamos, también, la relevancia que este estudio adquiere en la óptica de preservar signos importantes de la tradición artesanal. Hoy en día la preocupación de muchos (por ej. FUENTES, 2011) es que se va perdiendo la identidad cultural y la cercanía a los valores del pasado.

Hemos estudiado, también, el trabajo de Owen, (OWEN, 1995) para tomar como herramienta, en la parte de modelización, lo que él usa y que se adapta a nuestro objeto de estudio, como se expone en Albanese (ALBANESE, 2011).

Nuestro trabajo se desarrolla según una estructura parecida a la de las investigaciones apenas citadas en:

- la búsqueda de una metodología de análisis que se ajuste a un objeto de estudio específico;

- en dicha metodología la definición de fases (OLIVERAS, 1996), que aquí serán llamados factores, que ayudan a realizar el análisis, tanto 
etnográfico como matemático;

- la elección del objeto que sea un signo, una expresión relevante, o sea un artefacto característico de una microcultura.

$Y$ trata de conectar dos microculturas, como ya hemos indicado, partiendo de ejemplares encontrados en el contexto y de los aportes metodológicos de los antecedentes sobre los que hicimos un trabajo creativo de nuevo método, que pasamos a exponer.

\section{Diseño de la investigación y Método MOMET elaborado}

\subsection{Diseño y Método. El diseño de esta investigación es del siguiente tipo:}

- no experimental, porque no se interviene activamente, intencionalmente para modificar las situaciones observadas sino que se considera el entorno en su natural complejidad;

- también es trasversal, ya que los datos se consideran recogidos durante un mismo momento observatorio; y

- es exploratorio, ya que se trata de una primera aproximación mediante el estudio de varios casos.

Tal diseño constituye una estructura o formato adecuado para la idea pretendida de realizar una modelización de cierto signo del contexto de las artesanías en términos matemáticos formales.

Se elige una metodología etnográfica por su adecuación a los objetivos de la investigación y por la ventaja que este tipo de método de investigación proporciona, o sea, la flexibilidad y apertura que le otorga su orientación naturalista y fenomenológica, con la constante atención al contexto sociocultural que caracteriza la componente etnográfica de esta la investigación.

El instrumento metodológico MOMET que se crea para este estudio interpretativo formal de artesanías de trenzado tiene en cuenta dos aspectos:

$\checkmark$ el objeto o producto final de la labor artesanal, analizado en su complejidad global y

$\checkmark$ el proceso que se lleva a cabo para construir el objeto artesanal.

La idea es desarrollar un método para realizar la investigación desde el punto de vista etnográfico y, después, desde el punto de vista de la matemática formal (producto y proceso, respectivamente).

Esta herramienta metodológica que hemos elaborado está constituida, 
entonces, por dos componentes, por un Método de análisis ETnográfico (MET) y por un Modelo de análisis matemático o tipo de MOdelización Matemática (MOM). El conjunto de los dos nos proporciona el instrumento metodológico que denominamos MOMET.

Ponemos de manifiesto que, por su especificidad, a una definición teóricoconceptual del objeto de investigación, se prefiere una descripción operativa, o sea una caracterización del mismo a través de casos o ejemplos paradigmáticos concretos que indicaremos como ejemplares. Una recopilación de cordeles y trenzas permitirá, así, delimitar el contexto artesanal referente y construir, de manera inductiva y realística, una descripción del objeto, que valga como definición ejecutiva para los fines del estudio. Analizamos estos ejemplares mediante la aplicación del MOMET.

Entonces, como unidad de análisis se considera el ejemplar concreto y real tomado. Solo en un estadio posterior del análisis se identificarán patrones y se establecerán relaciones entre ejemplares.

\subsection{MET: método de análisis etnográfico}

Está compuesto por delimitación descriptiva de los factores clave emergentes del objeto situado en su entorno cultural, necesarios para definir dicho objeto y expresados en forma genérica aplicable a todos los ejemplares, de modo que tienen un carácter definitorio y modelizador.

Así que, empezamos describiendo los factores sobre los cuales se basa el estudio etnográfico para, posteriormente, indicar dónde y cómo interviene la modelización matemática.

Vamos a identificar estos factores claves de la metodología del análisis etnográfico (MET):

1. Factor de caracterización. Se refiere a la descripción, como forma de definición del objeto de estudio, es decir del ejemplar, y está constituido por tres variables:

a. Proveniencia histórico geográfica del ejemplar;

b. Rápida descripción o explicación verbal sucinta del mismo;

c. Imagen o representación visual estática (Figura) o dinámica (video) de él.

2. Factor utilidad. Se indica en este ángulo descriptivo:

a. Para qué acción (en la construcción, en la industria, en la agricultura, con animales, etc.) se utiliza y 
b. Donde (lugar geográfico o contexto macro: en Argentina, en la Pampa, etc.; o bien lugar social o contexto micro: la casa, el campo, el taller, etc.) cada ejemplar de cordel es utilizado.

3. Factor material. Se consideran varios aspectos de los materiales empleados en la construcción del objeto artesanal que se estudia, cordeles y trenzas en este caso:

a. Se considera la cualidad natural del material (por ejemplo cuero, algodón, lana, etc.) o naturaleza del material; y se describen aquellos materiales que se pueden emplear juntos en el mismo objeto;

b. Se estudia cómo se realiza la preparación de los materiales, o sea si hay unos procesos previos preparatorios del material: para teñir, cortar o ablandar los materiales, seleccionarlos, etc. antes de comenzar la tarea artesanal de trenzar.

c. Las propiedades físicas de los materiales, la resistencia, la flexibilidad, el peso, la maleabilidad, dureza, color, etc., que están muy relacionados con el factor cualidad o naturaleza de los materiales constitutivos.

4. Factor modalidad de tejido. Se analizan los tipos de tejido, o forma en que se mezclan las fibras, cabos o hilos:

a. Se distingue, en primer lugar, entre las labores de mezclar hilos que presentan nudos, o anudados (PARRA, 2003), y las que

b. No presentan nudos, o trenzados. La modalidad trenzado tiene la peculiaridad de que, en cualquier punto, si se deja sin atar la madeja se va soltando, se desarma, o sea se separan los cabos o hilos que la forman. Esto no ocurre en los anudados.

c. Una categoría emergente acá es la del cortado e insertado, o sea los ejemplares que presentan hilos que vienen cortados para que otros hilos puedan atravesarlos.

d. Otra categoría emergente es la que llamamos tejido, o sea cuando, además de no haber nudos, se puede distinguir entre trama y urdimbre (las fajas).

e. Las herramientas que se manejan para el tejido, si son las solas manos, o hay el uso de artefactos suplementarios como telares de varios tipos, (véanse el aparato llamado la carta, en la Figura 1). El uso de las manos, y, a veces, de los pies en los telares, interviene esencialmente en la definición de las artesanías.

Para la modelización matemática que sigue este factor es esencial: de aquí en adelante, o sea para el sucesivo o siguiente factor: el Diseño, se van a 
considerar solo los ejemplares cuya modalidad de tejido es el trenzado, ya que focalizamos el estudio en este tipo de objetos.

El uso de herramientas o aparatos puede intersecar con varias modalidades de tejido.

5. Factor diseño. Este es el factor que caracteriza el proceso de trenzar. Acá se consideran:

a. El número de hilos, donde por hilo se entiende el cabo, la unidad primordial de la fibra que se va trenzando;

b. El número de colores, si hay distintos, y cuantos hilos hay por cada color;

c. La forma predominante del ejemplar que se va tejiendo, o sea la visión global (BOLAÑOS, 2009) o de conjunto del objeto que se va produciendo (cuadrado, redondo, lineal, etc.);

d.La manera de trenzar, la secuencia de acciones que se tienen que cumplir para llegar a realizar el trenzado, el proceso dinámico de la acción artesanal de trenzar, propia del ejemplar.

e.La trama del trenzado, el producto desde el punto de vista estático, el esquema que configura ese objeto.

Otros factores a considerar en un análisis completo, pero que por razones de tiempo no desarrollamos en detalle para los ejemplares que tratamos en este trabajo, son:

6. Factor dimensión.

Ya hemos identificado las artesanías de trenzado como las artesanías cuyo producto está caracterizado por su desarrollo en una dimensión, digamos la longitud (o largueza), que predomina sobre las otras dos. Se consideran, entonces:

a. La extensión según esta dimensión predominante. Si son objetos cortos

o pequeños en esa dimensión o por el contrario son largos o grandes.

b. Relación de la extensión, con el grosor, el uso y el diseño de trenzado.

Se consideran también elementos relevantes, por razones sociales, los factores siguientes:

7. Factor tiempo.

a. Cuanto se tarda en trenzar una secuencia mínima, por un artesano experto;

b. El tiempo empleado en terminar un producto, o la durada del producto. 


\section{Factor económico.}

a. El coste del producto, neto, la ganancia esperada y el precio comercial (si hay precios establecidos), productos considerados caros o baratos.

b. Quien le pone el precio de venta, (el artesano/a o el comerciante si son diferentes personas) y sobre qué se realiza la estimación (consumo energético, materiales, tiempo empleado, producto deteriorado, etc.) $\mathrm{y}$

c. Cómo se estima el precio (con anotaciones, considerando facturas de gastos realizados, utilizando calculadora, teniendo en cuenta solo las necesidades de dinero inmediatas), por parte de los artesanos.

\subsection{MOM: Modelización Matemática}

La conexión entre los aspectos etnográficos y matemáticos que estudiamos en esta sección se realiza a nivel del factor 5 o diseño y concentrada en el proceso activo de trenzar. Vamos a desarrollar una modelización teórica que traduzca, en el lenguaje de la matemática formal, el diseño del trenzado, y, precisamente, a partir de la manera activa de realizar la acción de trenzar.

Realizamos el análisis en dos momentos, considerando primero el proceso según su desarrollo en sección horizontal, imaginando mirar la trenza o el cordel en construcción desde el punto de vista de la cola, o sea de donde los hilos están a punto de ser trenzados.

Después, observamos el recorrido de los hilos según la sección vertical, mirando el diseño en el producto ya terminado, según la dimensión longitudinal mayor.

\subsubsection{Sección Horizontal - Modelización con grafos}

El lenguaje de la matemática formal que utilizamos ahora en la modelización de la sección horizontal es el de la Teoría de Grafos.

Un grafo $|V|$ es un par ordenado $G=(V, E)$, donde $V$ es un conjunto de vértices o nodos, y $E$ es un conjunto de arcos o aristas, que relacionan estos nodos. Se considera finito y se llama orden de $G$ al número de vértices de $V$, indicado $|V|$.

Consideramos la definición de Chacón (2005) que, inspirándose en Brualdi (1997), dice: 
Un grafo $\mathrm{G}$ es un par $\mathrm{G}=(\mathrm{V}, \mathrm{E})$, donde $\mathrm{V}$ es un conjunto finito (vértices, nodos) y $\mathrm{E}$ es un multiconjunto de pares no ordenados de vértices, denotados por $\{\mathrm{x}, \mathrm{y}\}$, que se denominan lados, aristas, etc. En este caso decimos que $\mathrm{x}$, $y$ son extremos de $\{x, y\}$. Denotamos V(G) por el conjunto de vértices del grafo $\mathrm{G}$ y por $\mathrm{E}(\mathrm{G})$ el conjunto de lados del grafo $G$. Además $V(G)$ y $E(G)$ denotan el número de vértices y el número de aristas de $\mathrm{G}$ respectivamente.

Puesto que E es un multiconjunto es posible que existan pares repetidos, en este caso $\mathrm{G}$ tiene lados múltiples. También es posible que algún par no ordenado de $\mathrm{E}$ tenga el mismo vértice repetido, en este caso decimos que el lado es un lazo (loop) o bucle. Cuando existen lados múltiples y/ o lazos decimos que $\mathrm{G}$ es un multigrafo. Si no hay lados múltiples ni lazos decimos que es un grafo simple. Un digrafo $\mathrm{G}$ es un par $\mathrm{G}=(\mathrm{V}, \mathrm{E})$ donde $\mathrm{V}$ es un conjunto de vértices y $\mathrm{E}$ es un multiconjunto de pares ordenados. Los lados se denotan por pares ordenados, $(u, v)$ denota el lado dirigido que tiene como vértice inicial a $u$ y como vértice terminal a v. (CHACÓN, 2005, p. 1; itálico del autor).

Añadimos otra definición, de Rosen, que insiste en la idea importante de aristas múltiples y multígrafo, nuestra concepción de grafo reúne a las diferentes definiciones.

Un grafo simple $\mathrm{G}(\mathrm{V}, \mathrm{E})$ consta de $\mathrm{V}$, un conjunto no vacío de vértices, y de $\mathrm{E}$, un conjunto de pares no ordenados de elementos distintos de V. A esos pares se les llama aristas. [...]. Los grafos simples no bastan para modelar esta situación. En lugar de grafos simples, emplearemos multígrafos, que constan de vértices y de aristas no dirigidas entre estos vértices, pero admitiendo la existencia de aristas múltiples entre pares de vértices. [...] Esto hace que la definición de multígrafo es un poco más complicada. Un multígrafo $\mathrm{G}(\mathrm{V}, \mathrm{E})$ consta de un conjunto $\mathrm{V}$ de vértices, un conjunto $\mathrm{E}$ de aristas y una función $f$ de $\mathrm{E}$ en $\{\{u, v\} \mid u, v$ $\epsilon V, u \neq v\}$. Se dice que las aristas $e_{1}$ y $e_{2}$ son aristas múltiples o paralelas si $f\left(e_{1}\right)=f\left(e_{2}\right)$. [...]

Un grafo dirigido $(\mathrm{V}, \mathrm{E})$ consta de un conjunto $\mathrm{V}$ de vértices $\mathrm{y}$ de un conjunto $\mathrm{E}$ de aristas que son pares ordenados de elementos de V. (ROSEN, 2004, p. 504-505; itálico del autor).

En la modelización que presentamos, los vértices o nudos representan 
las posiciones de los hilos a punto de ser trenzados, los indicaremos con letras minúsculas. Los arcos o aristas representan los movimientos de los hilos, respecto a la posición, que el artesano tiene que hacer cumplir a los hilos para crear la trama.

Estudiamos la secuencia mínima de movimientos que se van repitiendo y que caracterizan, unívocamente, el trenzado.

Distinguimos varias fases, que denominamos movimiento mínimo, paso y secuencia simple o compuesta, y las modelizamos en dos momentos o situaciones: Sección horizontal y Sección Vertical, veamos la conceptualización de esto.

En la Sección horizontal definimos:

a. Movimiento mínimo: es el movimiento que involucra dos o más hilos que intercambian sus posiciones; el conjunto de hilos es el mínimo tal que cada cabo del conjunto, en su movimiento, vaya ocupando una posición dejada vacía por el movimiento de otro cabo del conjunto y, a su vez, deje una posición vacía que sea ocupada por otro cabo del conjunto. Se describe en el grafo a través de un circuito simple. Esta caracterizado por:

i. Cuantas y cuales posiciones se intercambian, o mejor dicho, lo que se intercambian son los hilos que se encuentran en determinadas posiciones. Aclaramos que, por razones de claridad y fluidez del discurso, de aquí en adelante con posiciones nos referimos a los hilos que se encuentran en las posiciones determinadas en el paso en cuestión.

ii. Un sentido horario o anti horario.

b. Paso: un paso del proceso de trenzar es el máximo conjunto de movimientos mínimos tal que cada vértice no pertenece a más de un movimiento. Un paso se representa en un único grafo en el que aparecen eventualmente más circuitos no conectados. Está caracterizado por

i. Números de movimientos mínimos que constituyen el paso.

ii. Orden de los movimientos mínimos.

c. Secuencia simple o compuesta: si la secuencia mínima se describe con un solo paso, es suficiente un solo grafo para describirla (simple); si la secuencia incluye más de un paso, se necesita más de un grafo para describirla (compuesta).

Señalamos que todos los grafos de cada paso tienen la misma estructura 
(o esqueleto), o sea, en términos técnicos, el grafo vacío asociado, cuyo conjunto de aristas es nulo, es el mismo. Esto significa que, si a cada grafo de cada paso le quitamos las aristas, obtenemos siempre el mismo grafo vacío, que acá llamamos grafo estructura. El grafo estructura está determinado por el diseño. Los grafos estructuras que consideramos son todos cuadrados, o sea los vértices o nudos se disponen sobre los lados de un cuadrado.

Observamos que en este estadio del análisis no nos interesan particularmente los colores de los hilos, sino cómo se disponen los hilos, si son de distintos colores, en el momento de iniciar el trabajo, influye mucho sobre la apariencia final del cordel. Así que, cuando vayamos a analizar ejemplares concretos constituidos con hilos de dos o más colores, damos la disposición inicial de los hilos, según los colores, en el grafo estructura.

Para aclarar el concepto, hacemos un ejemplo con la trenza simple de tres hilos, la clásica trenza del pelo, en el párrafo siguiente.

\subsubsection{Sección Vertical - Modelización combinatoria}

Ahora vamos a utilizar el lenguaje de la combinatoria como lenguaje de la matemática formal para seguir modelizando el proceso de trenzar. Dado un conjunto finito de elementos, llamado $V$, una permutación es una correspondencia (o aplicación) biyectiva de $V$ en sí mismo, $p: V \rightarrow V$, a veces indicada como reordenamiento. El conjunto de las permutaciones en $V$ con la operación de composición forma un grupo, indicado $S_{V}$.

Se llama ciclo, y se indica $\sigma=\left(x_{1}, x_{2}, \ldots, x_{n}\right)$, la permutación que manda cíclicamente cada elemento en su sucesivo, o sea $x_{i}$ en $x_{i+1}$ hasta $x_{n}$ en $x_{1}$, mientras deja fijos los que no aparecen. Si el ciclo contiene solo dos elementos se llama transposición. Dos ciclos se dicen disjuntos si no comparten ningún elemento de $V$. Cada elemento del grupo de permutaciones se puede escribir como composición de ciclos disjuntos (la composición, si los ciclos son disjuntos, es simplemente una yuxtaposición). Así que, para expresar las permutaciones vamos a utilizar la notación de composición de ciclos disjuntos.

Volvemos a las trenzas. Los grafos utilizados en la sección anterior nos permiten detectar de qué manera se realiza la acción de trenzar en función de una posición inicial de los hilos y de una secuencia de intercambios de estas posiciones. Esta misma secuencia se puede presentar a través de la combinatoria expresando los movimientos mínimos, o sea los circuitos, como ciclos del grupo de permutaciones $S_{V}$ (donde $V$ es el conjunto de nudos del grafo estructura) y 
describiendo los pasos con otros elementos del grupo de permutaciones que salen de la composición de estos ciclos.

Se observa que, de esta manera, no se incluye la información sobre la colocación de las posiciones, o sea el grafo estructura, y se puede perder la información sobre el sentido, (horario o anti horario), del circuito que involucra solo dos posiciones.

En analogía a lo visto para la modelización con grafos, ahora se encuentran las mismas fases.

a. Movimiento mínimo: ya hemos visto que a cada circuito del grafo se asocia un ciclo, caracterizado por:

i. Cuantas y cuales posiciones se cambian.

ii. El sentido. Vamos aclarando que si el ciclo está compuesto por más de dos elementos, $\left(x_{1}, x_{2}, \ldots, x_{n}\right)$, el sentido del circuito asociado es de $x_{1}$ a $x_{2}, \ldots$, hasta $x_{n}$; esto implica que el sentido queda unívocamente determinado por la escritura del ciclo, una vez noto el grafo estructura, o sea la ubicación de los vértices o nudos en el espacio, (el circuito queda horario o anti horario según como se diseñan los vértices en el grafo de base que he llamado grafo estructura).

Si el ciclo es una transposición, asumimos la siguiente convención: si suponemos que $x_{1}<x_{2}$ (en el ordenamiento alfabético), entonces un circuito entre $x_{1}, x_{2}$ en sentido horario será $\left(x_{1}, x_{2}\right)$; un circuito $x_{1}, x_{2}$ en sentido anti horario será $\left(x_{1}, x_{2}\right)$.

b. Paso: se representa con un elemento del grupo $S_{V}$ que resulta, eventualmente, de la composición de más de un ciclo. Se caracteriza por:

iii. Número de ciclos que lo constituyen.

iv. Se considera el orden en el que aparecen escritos los ciclos como el orden de ejecución de los movimientos.

c. Secuencia simple o compuesta: si la secuencia mínima se describe con un solo paso, es suficiente una sola permutación para describirla (simple); si la secuencia incluye más de un paso, se necesita más de una permutación (compuesta). 


\section{Caso de la trenza simple de tres hilos}

Aplicando el MOMET hacemos un ejemplo con la trenza simple de tres hilos, la clásica trenza del pelo.

\subsection{Sección horizontal: grafos}

Se trata de una secuencia compuesta, en particular está formada por dos pasos, así que se necesitan dos grafos para describirla. Llamamos a las posiciones de los hilos con las letras minúsculas $a, b, c$. En este caso, los nudos los visualizamos así: $a$ sobre el lado horizontal arriba del cuadrado, $b$ sobre el lado vertical de la derecha y $c$ sobre el lado vertical de la izquierda.

Los dos pasos de la secuencia son, en el orden siguiente: el primero constituido por un circuito simple horario entre las posiciones $a$ y $b$; el segundo constituido por un circuito simple anti horario entre las posiciones $a$ y $c$. La aclaración del punto (a.i.), del párrafo anterior, significa que las letras no se mueven, o sea, quedan asociadas a la posición, así que, en pasos sucesivos, siguen refiriéndose al mismo nudo del grafo estructura asociado al diseño (Figura $6)$.

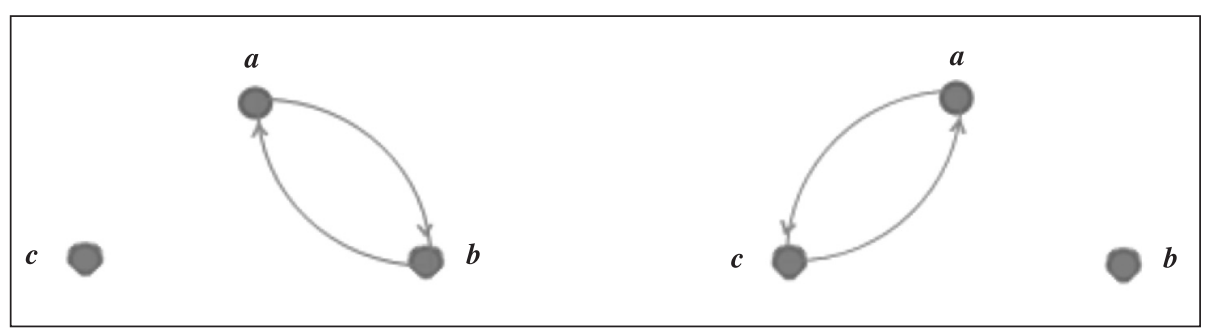

Figura 6 - Grafos de la trenzas simple

\subsection{Sección vertical: combinatoria}

En el ejemplo que estamos analizando, de la trenza simple de tres hilos, el proceso de Modelización Vertical se describe a través de una secuencia compuesta de dos permutaciones de $S_{\{a, b, c\}}$ cada una constituida por una sola transposición;

$$
\begin{aligned}
& p_{1}=(a, b), \\
& p_{2}=(a, c) .
\end{aligned}
$$

De aquí en adelante, utilizamos la notación $p_{i}$ para los pasos o 
permutaciones, y $\sigma_{i j}$ para los ciclos o movimientos mínimos de $p_{i}$, donde el primer índice indica el paso de pertenencia y el segundo el orden de los ciclos en la permutación que representa el paso. Cuando el paso $p_{i}$ está constituido por un solo ciclo, indicamos el ciclo directamente con $p_{i}$. Se observa que, en este caso de la trenza simple, siendo $p_{2}$ una trasposición con cambio anti horarios, la letras están en orden (alfabético) decreciente.

Recordamos que, en cada paso, las letras siguen asociadas a las mismas posiciones, los que se mueven son los hilos.

Ahora, ponemos de manifiesto las razones por las cuales este segundo análisis ha sido nombrado como Sección Vertical. Si numeramos los hilos, asignándoles los números naturales $1,2, \ldots,|V|$, es posibles seguir el recorrido de los hilos en la trama de la trenza según la sección vertical. Para ejecutar el cambio de posiciones de los hilos que se realiza durante un paso, se aplica a los números la permutación que representa el paso. Vamos a explicar cómo: la permutación que caracteriza el paso es expresada en función de las letras que indican las posiciones, así que, en realidad, para describir un determinado paso se tiene que cruzar la permutación con la información sobre las posiciones en las cuales se encuentran los hilos justo antes de realizarlo. Se genera, así, una nueva permutación, esta vez en función de los números que indican los hilos, sustituyendo a cada letra el número del cabo que en ese momento ocupa la posición indicada por la letra.

Realizamos este procedimiento en una tabla. Las primeras $|V|$ columnas son las posiciones, así que en las primeras $|V|$ celdas de cada línea se ponen los números de los hilos que ocupan las correspondientes posiciones. La $|V|+1$ columna contiene la permutación que caracteriza el paso a aplicar a la configuración descrita en las anteriores columnas en términos de las posiciones. La última columna representa la permutación para aplicar a la configuración en función de los números de los hilos. La tabla termina cuando se consigue de vuelta la configuración inicial.

Sigue la tabla T1 de la Trenza simple de 3 cabos, que hemos llamado del pelo: 
Tabla T1 - de la Trenza simple de 3 cabos (Tabla de datos de elaboración propia)

\begin{tabular}{|c|c|c|c|c|}
\hline $\boldsymbol{a}$ & $\boldsymbol{b}$ & $\boldsymbol{c}$ & $\boldsymbol{p}_{\boldsymbol{i}}$ & Paso especifico \\
\hline 1 & 2 & 3 & $p_{1}=(a, b)$ & $(1,2)$ \\
\hline 2 & 1 & 3 & $p_{2}=(a, c)$ & $(2,3)$ \\
\hline 3 & 1 & 2 & $p_{1}=(a, b)$ & $(3,1)$ \\
\hline 1 & 3 & 2 & $p_{2}=(a, c)$ & $(1,2)$ \\
\hline 2 & 3 & 1 & $p_{1}=(a, b)$ & $(2,3)$ \\
\hline 3 & 2 & 1 & $p_{2}=(a, c)$ & $(3,1)$ \\
\hline 1 & 2 & 3 & - & - \\
\hline
\end{tabular}

\section{Conclusiones}

En nuestra investigación, por su enfoque metodológico y su objetivo, las conclusiones son fruto de una introspección final sobre algunos puntos clave que se han aclarado con lo aportado mediante el trabajo de investigación.

Tras la reflexión y un posterior debate se llegó a las conclusiones siguientes:

a) El artesano tiene que ser capaz, en cada momento, de reconocer en qué punto se encuentra, entonces registra este recorrido, aunque no conscientemente, o con metaconocimiento de lo que está haciendo, sino en forma de Rutina y no representa sus acciones en estos términos formales.

b) Se puede entender o definir el cordel ejemplar como representante de una clase de equivalencia determinada por un cierto diseño, en la que existen muchos cordeles, que pertenecen a distintas artesanías, pero a efectos del trenzado, visto matemáticamente, son equivalentes. Tomamos uno como representante, para analizarlo y poder definir $l a$ clase en términos matemáticos, diciendo que todos los cordeles que tienen esa modelización son equivalentes. Etnográficamente, puede que no sean equivalentes para el uso social, aunque tengan igual tipo de trenzado, si cambia el material, por ejemplo el cuero por algodón o por seda. Por eso la unidad de análisis matemático es el ejemplar, que luego se generaliza al modelizarla, y se particulariza mediante los otros factores que no son su estructura matemática, pero que son esenciales para una artesanía.

c) Una artesanía puede tener ejemplares no equivalentes, es decir con distintos tipos de trenzados. Pero esto hay que definirlo y fundamentarlo 
en el seguimiento de esta investigación, encontrando:

1- una definición social de cada artesanía,

2- ejemplares diversos matemáticamente de cada artesanía,

3- ejemplares de diferentes artesanías y sus modelos matemáticos,

4- Agrupamientos de los ejemplares por su modelo y por su artesanía.

5- Un método para la modelización de ejemplares cualesquiera.

d) Dentro de lo que se ha podido encontrar en la primera fase del trabajo de campo, por ahora, hablamos solo de ejemplares concretos y de artesanía de trenzado en general, sin pretender distinguir entre diferentes artesanías, que se estudiarán en la continuación de esta investigación, en la tesis doctoral.

Entre las reflexiones finales o hallazgos queremos incluir el gran impacto personal sentido con esta investigación por las dos investigadoras, los aprendizajes personales realizados durante el proceso de la investigación, que como investigación Etno-Matemática ha involucrado globalmente sus vidas, no solo su intelecto.

Y, especialmente, queremos mostrar nuestro agradecimiento y rendir un homenaje a los artesanos y artesanas, que mantienen vivas las matemáticas insertadas de forma implícita en los objetos culturales, que ellos hacen con tanto conocimiento y esfuerzo y que enseñan a hacer con tanta generosidad.

Esta investigación está soportada por una Beca FPU (código de referencia AP2010-0235) del Ministerio de Educación, Cultura y Deporte del Gobierno de España, concedida a la investigadora V. Albanese.

\section{Referencias}

ALBANESE, V. Etnomatemáticas en Artesanías de Trenzado. 2011. 73f. Tesis (Máster en Didáctica de la Matemática) - Facultad de Educación, Departamento de Didáctica de la Matemática, Universidad de Granada, Granada, España, 2011.

ASCHER, M.; ASCHER R. Code of the Quipu: a study in media, mathematics and culture. Ann Arbor, MI: The University of Michigan Press, 1981.

ASCHER, M. Ethnomathematics, a multicultural view of mathematical idea. California: Pacific Grove, Brooks \& Colé, 1991.

BARTON, B. Making sense of ethnomathematics: Ethnomathematics is making sense. Educational Studies in Mathematics, Hidelberg (Germany), v. 31, n. 1, p. 201-233, Sep. 1996. 
BISHOP, A. Mathematical enculturation: A cultural perspective on mathematics education. Dordrecht, Holland: Kluwer Academic Pub, 1991.

BOLAÑOS, J. Una visión etnomatemática de las pintaderas canarias. 2009. 104f. Tesis (Máster en Didáctica de la Matemática) - Facultad de Educación, Departamento de Didáctica de la Matemática, Universidad de Granada, Granada, España, 2009.

BOLÍVAR, A. Ciudadanía y escuela pública en el contexto de diversidad cultural. Revista Mexicana de Investigación Educativa. México D.F. v. 9, n. 20, p. 15-38, jul./ sept. 2004.

BORBA, M. Ethnomahtematics and Education. For the Learning of Mathematics, Fredericton (Canada), v. 10, n. 1, p. 39-43, Jan. 1990.

BRUALDI, R. A. Introductory Combinatorics. North-Holland: Elsevier, 1997.

CHACÓN, J.L. Introducción a la Teoría de Grafos. Matemática Discreta. 2005. Disponible en: http://webdelprofesor.ula.ve/ciencias/jlchacon/materias/discreta/ grafos.pdf. Acceso el: 29 oct. 2012.

DA COSTA, L. M. Los tejidos y las tramas matemáticas. El tejido ticuna como soporte para la enseñanza de las matemáticas. 2009. 184f. Tesis (Maestría en Estudios Amazónicos) - Universidad Nacional de Colombia, Amazonia, 2009.

DE BENGOECHEA, N. Etnomatemáticas, métodos y objetos culturales. 2009. 85f. Tesis (Máster en Didáctica de la Matemática) - Facultad de Educación, Departamento de Didáctica de la Matemática, Universidad de Granada, Granada, España, 2009.

DE LOS SANTOS, A. M. Artesanías con cuero. Buenos Aires: Grulla, 2004.

D'AMBROSIO, U. Ethnomathematics and its place in the history and pedagogy of mathematics. For the learning of Mathematics, Fredericton (Canada), v. 5, n. 1, p. 4448, Jan. 1985.

D’AMBROSIO, U. Etnomatemática: Eslabón entre las tradiciones y la modernidad. México: Limusa, 2008.

EGLASH, R. When Math Worlds Collide: Intention and Invention in Ethnomathematics. Science, Technology, \& Human Values, Pittsburgh (U.S.A.), v. 22, n. 1, p. 79-97, Jan. 1997.

ESCUDERO, J. M.; GONZÁLEZ, M. T.; MARTÍNEZ, B. El fracaso escolar como exclusión educativa: comprensión, políticas y prácticas. Revista iberoamericana de educación, Madrid, v. 50, n. 2, p. 41-64, mayo/ago. 2009. 
FAUDONE, H. EL arte gaucho del cuero crudo. Valencia: Editora Valencia, 2003.

FIADONE, A. El diseño indígena argentino. Buenos Aires: la Marca editora, 2003.

FLORES, L. A. El guasquero: trenzados criollos. Buenos Aires: Cesarini Hermanos, 1960.

FONTANA, A. La artesanía tradicional del cuero en la Mesopotamia Argentina. Paraná, Argentina: Editorial Entre Ríos, 1988.

FUENTES, C. C. Algunos Procedimientos y Estrategias Geométricas Utilizadas por un Grupo de Artesanos del Municipio de Guacamayas en Boyacá, Colombia. Revista Latinoamericana de Etnomatemática, Pasto (Colombia), v.4, n.1, p. 55-67, jan. 2011.

GERDES, P. On culture and mathematics teacher education. Journal of Mathematics Teacher Education, Hidelberg (Germany), v. 1, n. 1, p. 33-53, Jan. 1998.

MICROSOFT ENCARTA. Enciclopedia Encarta. Washington: Microsoft Corporation. 2009. DVD

OLIVERAS, M. L. Etnomatemáticas. Formación de profesores e innovación curricular. Granada: Comares, 1996.

OLIVERAS, M. L. Mathematics and craftwork in Andalusia an antropological-didactic study. Isgem Newsletter, Syracuse (USA), v. 13, n.1, p. 3-5, Nov, 1997.

OLIVERAS, M. L.; FERNÁNDEZ, J.; FUENTES, J. Etnomatemáticas y educación Matemática. Construyendo un Futuro Equitativo. In: PRIMER CONGRESO INTERNACIONAL DE ETNOMATEMÁTICAS, 1., ICEm, 1998, Granada. Actas... Granada, Universidad de Granada. 1998. CD-ROM.

OLIVERAS, M. L. Ethnomathematics and Mathematical Education. International Reviews on Mathematical Education, Jahrgang, v. 3, n. 3, p. 85-91, June 1999.

OLIVERAS, M. L. Etnomatemáticas. De la multiculturalidad al mestizaje. En: GIMÉNEZ, J.; GOÑI J. M.; GUERRERO S. (Eds.) Matemáticas e interculturalidad. Barcelona: Graó, 2006, p.117-149.

OLIVERAS, M. L. Model for Research on Multiculturality in Mathematics Education. In: INTERNATIONAL CONGRESS ON MATHEMATICAL EDUCATION -ICME, $11^{\text {th }}$, 2008, Monterrey. Proceedings... Monterrey, 2008a. p.1-23. Topic Study Group 33: mathematics education in a multilingual and multicultural environment. 
OLIVERAS, M. L. The IDMAMIM Project is "Innovation in Didactics for Mathematics in Multicultural contexts, with Immigrant and Minority pupils". In: INTERNATIONALCONGRESS ON MATHEMATICALEDUCATION-ICME, $11^{\text {th }}$, 2008, Monterrey. Proceedings... Monterrey, 2008b. Topic Study Group 33: mathematics education in a multilingual and multicultural environment.

OSORNIO, M. Trenzas gauchas. Buenos Aires: Hemisferio Sur, 1934.

OWEN, R. Braids: 250 patterns from Japan, Peru \& beyond. Loveland Colo: Interweave Press, 1995.

PARRA, A. Acercamiento a la Etnomatemática. 2003. 156f. Tesis (Licenciatura en Matemática) - Departamento de Matemáticas, Universidad Nacional de Colombia, Bogotá, 2003.

QUINTANILLA, M. A. Diccionario de filosofía contemporánea. Salamanca: Editorial Sígueme, 1976.

ROSEN, K.H. Matemática Discreta y sus aplicaciones. 5. ed. Madrid: McGraw-Hill Interamericana, 2004.

SANDELLA, O. La geometría en las danzas folklóricas argentinas. En: DÍAZ, L. (Ed.) Acta Latinoamericana de Matemática Educativa vol. 17, Buenos Aires, Comité Latinoamericano de Matemática Educativa, 2004, p. 801-806.

SANTILLÁN, A.; ZACHMAN, P. Una experiencia de capacitación en Etnomatemática. Revista Latinoamericana de Etnomatemática, Pasto (Colombia), v. 2, n. 1, p. 27-42, jan.-jul. 2009.

SERVETTO, L.; CASTILLA, C.; NAVARRO M.; VAQUERO, A. La artesanía en la zona andina argentina. Córdoba: Universidad de Córdoba, 1998.

TEDESCO, J. C. Los desafíos de la educación básica en el siglo XXI. Revista Iberoamericana de Educación. Madrid, n. 55, p. 31-47, jan.-abr. 2011.

UÑA, O; HERNÁNDEZ, A. Diccionario de Sociología. Madrid: Editorial ESIC, 2004.

VILELA, D. S. Discussing a philosophical background for the ethnomathematical program. Educational Studies in Mathematics, Hidelberg (Germany), v. 75, n. 3, p. 345358, Dec. 2010. 